\title{
Effects of Mutagens on Morphological traits of Psoralea corylifolia-A Medicinally Important Plant
}

\author{
*Ajey Karan Chaudhari ${ }^{1}$, Anand Prakash Singh² and B R Chaudhary ${ }^{3}$
}

\author{
${ }^{1}$ Department of Botany, \\ Paliwal P.G. College, \\ SHIKOHABAD, FIROZABAD \\ ${ }^{2}$ Department of Botany, \\ Bipin Bihari College, JHANSI \\ ${ }^{3}$ Department of Botany, \\ Institute of Sciences, Banaras Hindu University, \\ VARANASI \\ *Corresponding Author \\ E-mail: ajeykaran@gmail.com
}

Received : 18.08.2020; Accepted : 22.09.2020

\begin{abstract}
Mutation breeding like in other plants can significantly strengthen medicinal plants breeding programs and help to produce novel varieties with higher yield potential and improved yield quality. The dry and healthy seeds of $P$. corylifolia IC 111228 were subjected to mutagenic treatments namely ethyl methane sulphonate (EMS) and sodium azide (SA). The treatment concentrations $15 \mathrm{mM}, 30 \mathrm{mM}, 45 \mathrm{mM}$ and $60 \mathrm{mM}$ of EMS and $1 \mathrm{mM}, 2 \mathrm{mM}, 3 \mathrm{mM}$ and $4 \mathrm{mM}$ of SA were chosen to evaluate the mutagenic potential in either case. The morphological traits were evaluated in $\mathrm{M}_{1}$ generation viz. plant height, days to flowering initiation, seed yield/plant and 100-seed weight. In EMS treatment $15 \mathrm{mM}$ increased the plant height and seed yield, while in SA treatment $4 \mathrm{mM}$ increased the seed yield/plant and 100 -seed yield.
\end{abstract}

Figure : 01

Table : 01

KEY WORDS : Ethyl methane sulphonate, Mutation, Psoralea corylifolia, Sodium azide.

\section{Introduction}

The methods of plant breeding have become increasingly sophisticated since the days of simple selection method of vigorous plants among natural populations consisting of a mixture of parental genotypes and new having arisen as a result of various cytogenetic processes including genetic recombination known to occur in nature. Modern day plant breeding is based on creating variations and making selections, evaluation and multiplication of the desired genotypes. Mutagenesis is highly instrumental in plant biology for inducing genetic variability, in a great number of crops, mainly due to the fact that the technology is simple, relatively cheap to perform, applicable to all plant species and equally usable on small and large scale ${ }^{7,8}$. There are more than 120 species of $P$ soralea found worldwide, of which Psoralea corylifolia is widely used in the traditional system of remedies. $P$. corylifolia belongs to the family Fabaceae (chromosome no. $2 n=22$ ). It is commonly known as Babchi in Hindi. In India, it is found in Rajasthan and the eastern districts of Punjab, Uttar Pradesh and
Chhattisgarh. The seed-oil is used externally for the treatment of leucoderma, psoriasis and leprosy in Indian folk medicine ${ }^{4}$. It has potential to cure immune disorders ${ }^{2}$, and also has been noted as an anticancerous agent against leukemia and other cancer lines ${ }^{5,9,11}$. Additionally, this plant has antifungal activity against dermatophytic fungi such as Trichophyton rubrum and Trichophyton mentagrophytes ${ }^{5}$. Mutation breeding like in other plants can significantly strengthen medicinal plants breeding programs and help produce novel varieties with higher yield potential and improved yield quality of the $P$. corylifolia.

\section{Materials and Methods}

Seeds of $P$. corylifolia IC 111228 procured from National Bureau of Plant Genetic Resources (NBPGR), New Delhi, were used for induction of mutagenesis. Two chemical mutagens, namely ethyl methane sulphonate (EMS) and sodium azide (SA) were selected for this study.

Dry, healthy and uniform seeds of the $P$. corylifolia 
IC 111228 were soaked in distilled water for 15 hours. The seeds were then blotted and exposed to room temperature $\left(28 \pm 1^{\circ} \mathrm{C}\right)$ for removing surface water from the seeds. The pre-soaked seeds were treated at room temperature with the above chosen concentrations of EMS and sodium azide $\left(\mathrm{NaN}_{3}\right)$ for 4 and 8 hours durations. The treatment concentrations $15 \mathrm{mM}, 30 \mathrm{mM}, 45 \mathrm{mM}$ and $60 \mathrm{mM}$ of EMS and $1 \mathrm{mM}, 2 \mathrm{mM}, 3 \mathrm{mM}$ and $4 \mathrm{mM}$ of SA were chosen to evaluate the mutagenic potential in either case. The seeds were directly sown in the plots following a randomized block design (RBD) in triplicate containing with control. The following parameters were recorded in $M_{1}$ generations for assessment of morphological traits viz. plant height, number of primary branches, leaf area, internode distance, flowering time, spike length, number of seeds per spike, seed yield/plant and 100-seed weight.

\section{Results and Discussion}

The data related to mean and coefficient of variation for morphological traits in treated as well as control populations of $P$. corylifolia IC 111228 in $\mathrm{M}_{1}$ generation are presented in Table 1. The growing plants of control and treated were well flourished in $\mathrm{M}_{1}$ generation (Figure 1). The reduction in leaf area was maximum in $M_{1}$ generation reaching to $79.62 \pm 2.23 \mathrm{~cm}^{2}$ with $60 \mathrm{mM} \mathrm{EMS}$ applied for 8 hours, whereas maximum shift towards increase in leaf area was $120.74 \pm 4.20 \mathrm{~cm}^{2}$ with $15 \mathrm{mM}$ EMS administered for 4 hours. In $M_{1}$ generation, both the mutagens showed a progressive decrease in leaf area at all the treatment doses except $15 \mathrm{mM} \mathrm{EMS} \mathrm{for} 4$ hours. A significant increase or decrease in plant height was observed with most of the doses of EMS and SA in $M_{1}$ generation plants. In $M_{1}$ generation maximum increase in height $(187.35 \pm 3.14 \mathrm{~cm})$ was observed with $15 \mathrm{mM}$ EMS applied for 4 hours. In response to SA treatment a significant increase $(38.10 \pm 1.17)$ in the mean values of primary branches in $\mathrm{M}_{1}$ generation was observed with 4 $\mathrm{mM}$ applied for 4 hours. It is evident from the Table- 1 that decrease in internodal length was a common phenomenon in $\mathrm{M}_{1}$ generation plants with increase in concentration of both the mutagens. Contrarily, with EMS treatment a shift towards maximum increase in mean values of internode distance $(4.53 \pm 0.20 \mathrm{~cm})$ was observed in $\mathrm{M}_{1}$ generation with $15 \mathrm{mM}$ EMS for 4 hours.

In $M_{1}$ generation, EMS treated population with 45 $\mathrm{mM}$ for 4 hours showed significant effect on early initiation of flowering (53.90 \pm 1.84$)$, while late flowering initiation $(83.90 \pm 1.23)$ was observed in SA treated population with $4 \mathrm{mM}$ for 8 hours. Days to $50 \%$ flowering therefore, significantly increased or decreased in EMS and SA treated populations over control in both the generations. A significant reduction in the mean values for spike length except with $15 \mathrm{mM}$ EMS treated population was recorded in both the generations with all the treatments. In $\mathrm{M}_{1}$ generation, EMS treated population showed greater extent of variability with maximum increase in number of seeds per spike $(23.30 \pm 0.99)$ with $15 \mathrm{mM}$ EMS for 4 hours and maximum decrease in number of seeds per spike $(14.00 \pm 1.17)$ with $60 \mathrm{mM}$ for 8 hours. The most useful treatment was $4 \mathrm{mM} \mathrm{SA}$ for 4 hours, as it maximally enhanced the total yield per plant $(283.58 \pm 21.67 \mathrm{~g})$ in $\mathrm{M}_{1}$ generation, compared with control $(217.90 \pm 9.07 \mathrm{~g})$. The results obtained showed that a considerable increase in average values for 100 -seed weight was recorded in few treated populations of both mutagens in $\mathrm{M}_{1}$ generation. The 100-seed weight in $M_{1}$ population revealed significantly increased maximum $1.99 \pm 0.06 \mathrm{~g}$ with $60 \mathrm{mM}$ for 8 hours EMS treatment, while maximum reduction in mean value $1.39 \pm 0.03 \mathrm{~g}$ was recorded with $4 \mathrm{mM} \mathrm{SA}$ administered for 8 hours, compared with mean value $1.50 \pm 0.02 \mathrm{~g}$ for control.

It has been demonstrated that genetic variability for several desired characters can be induced successfully through mutations and its practical value in plant improvement programs has been well established. A number of workers ${ }^{1,3,10}$ have reported the role of chemical mutagens in enhancing genetic variability in higher plants. Genetic variability is the fundamental to successful breeding programs in both vegetatively and sexually propagated plants. Majority of the mutagenic treatments caused negative shift in the mean away from controls for yield and yield components in $M_{1}$ generations. Only a few treatments induced significant positive shift in the mean in $M_{1}$ generations. In the present study, some treatments showed slight increase or decrease in the mean values of different characters. The experimental findings under reference suggested that the mutagen induced variability measured as coefficient of variation (CV) normally increased in the treated populations as compared to their respective controls for all the polygenic characters of $P$. corylifolia IC 111228. All the administered doses of SA reduced plant height in M1 generation over control with maximum decrease at $4 \mathrm{mM}$ dose applied for 8 hours. Almost all the EMS treatments showed dose dependent decrease with the increasing concentrations and durations, except with the lowest dose which showed significant increase in plant height at $15 \mathrm{mM}$ EMS applied for 4 hours in $\mathrm{M}_{1}$ generations. It was also noted that the relationship between increase in variability and the corresponding doses administered was not linear. That might be due to DNA repair mechanism and natural selection process that reducing the number of variants/mutants and expected level of genetic variability.

\section{Conclusion}

The present experiment showed that in the case of SA treatment, $4 \mathrm{mM}$ concentration applied for 4 hours 


\begin{tabular}{|c|c|c|c|c|c|c|c|c|c|c|c|c|c|c|c|c|c|c|c|}
\hline \multirow{2}{*}{ 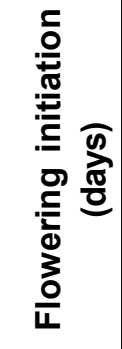 } & \multirow[b]{2}{*}{ م } & वे & $\underset{\forall}{\stackrel{\sim}{*}}$ & $\underset{\kappa}{i}$ & $\begin{array}{l}\qquad 0 \\
\infty \\
\infty\end{array}$ & $\begin{array}{l}\circ \\
\infty \\
\stackrel{0}{\circ}\end{array}$ & $\underset{i}{\tau}$ & $\begin{array}{l}\text { Oे } \\
\text { i. }\end{array}$ & $\begin{array}{l}\infty \\
\tilde{m} \\
\end{array}$ & $\stackrel{\infty}{\stackrel{\infty}{\infty}}$ & مْ & $\stackrel{\circ}{i}$ & 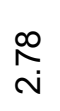 & $\stackrel{\widehat{o}}{\leftrightarrow}$ & "i⿱ & ๙ે & $\stackrel{\llcorner}{m}$ & $\begin{array}{l}\forall \\
\infty \\
\forall \\
\forall\end{array}$ & $\begin{array}{l}\stackrel{\leftrightarrow}{\emptyset} \\
\dot{\forall}\end{array}$ \\
\hline & & $\begin{array}{l}\frac{\complement}{\pi} \\
\sum \\
\sum\end{array}$ & $\begin{array}{l}\text { 8 } \\
0 \\
\text { +1 } \\
\text { o } \\
\text { ه }\end{array}$ & 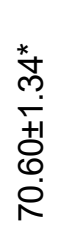 & 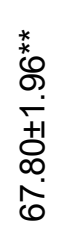 & 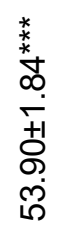 & 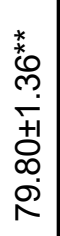 & $\begin{array}{l}* \\
0 \\
0 \\
0 \\
0 \\
+1 \\
0 \\
0 \\
0 \\
0\end{array}$ & 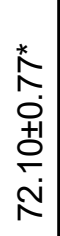 & \begin{tabular}{l}
0 \\
$\infty$ \\
0 \\
+1 \\
0 \\
\multirow{N}{N}{}
\end{tabular} & 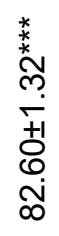 & 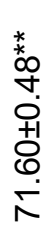 & 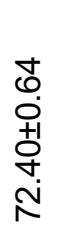 & 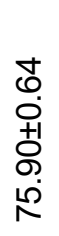 & $\begin{array}{l}\stackrel{m}{m} \\
\stackrel{+}{+1} \\
\stackrel{0}{0} \\
0 \\
0\end{array}$ & 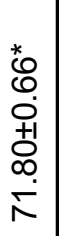 & 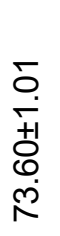 & 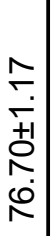 & 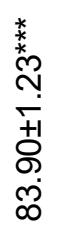 \\
\hline \multirow[b]{2}{*}{ 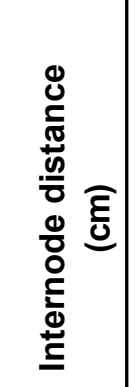 } & \multirow[b]{2}{*}{$\nabla$} & गे & $\begin{array}{l}\text { \& } \\
\text { ㅇ }\end{array}$ & $\begin{array}{l}\mathscr{8} \\
\stackrel{9}{\leftarrow} \\
\end{array}$ & $\begin{array}{l}\text { గิ } \\
\text { مْ }\end{array}$ & $\begin{array}{l}8 \\
\infty \\
\infty\end{array}$ & $\begin{array}{l}\infty \\
o \\
\dot{\square}\end{array}$ & $\underset{\text { N̦ }}{\hat{N}}$ & $\begin{array}{l}\infty \\
\stackrel{\infty}{\infty} \\
\stackrel{2}{\sim}\end{array}$ & $\stackrel{\bar{\kappa}}{\stackrel{m}{\Gamma}}$ & $\begin{array}{l}\text { ఠั } \\
\stackrel{0}{\Gamma}\end{array}$ & 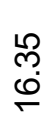 & $\begin{array}{l}80 \\
\dot{6}\end{array}$ & $\begin{array}{l}\hat{\emptyset} \\
\omega\end{array}$ & $\begin{array}{l}\hat{N} \\
\text { مீ }\end{array}$ & 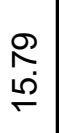 & $\frac{\omega}{\frac{10}{6}}$ & $\begin{array}{l}R \\
\stackrel{R}{N}\end{array}$ & $\begin{array}{l}\bar{n} \\
\text { مึ }\end{array}$ \\
\hline & & $\begin{array}{l}\frac{\Sigma}{\mathbb{J}} \\
\stackrel{\Phi}{\Sigma}\end{array}$ & $\begin{array}{l}\stackrel{n}{\circ} \\
\stackrel{+}{+} \\
\text { N̦} \\
\stackrel{+}{+}\end{array}$ & 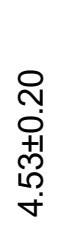 & 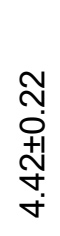 & 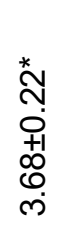 & 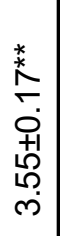 & 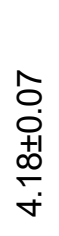 & $\begin{array}{l}\infty \\
\check{o} \\
+1 \\
\stackrel{+}{0} \\
\stackrel{+}{+}\end{array}$ & 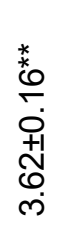 & 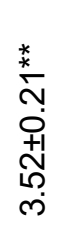 & 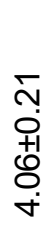 & 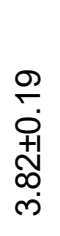 & 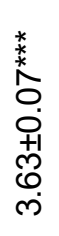 & 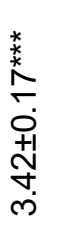 & 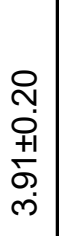 & 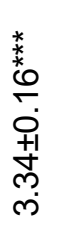 & 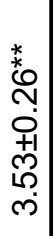 & 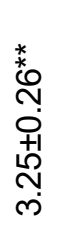 \\
\hline \multirow{2}{*}{ 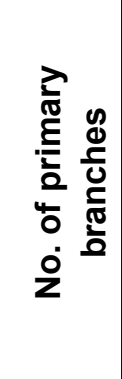 } & \multirow[b]{2}{*}{$m$} & गे & ஓ. & $\begin{array}{l}\hat{m} \\
\stackrel{\sim}{\sim}\end{array}$ & $\begin{array}{l}\infty \\
\infty \\
\dot{\square}\end{array}$ & $\begin{array}{l}\stackrel{0}{Ð} \\
\stackrel{\text { }}{\leftarrow}\end{array}$ & $\begin{array}{l}\stackrel{\mathscr{n}}{\mathrm{N}} \\
\stackrel{\mathrm{v}}{\sim}\end{array}$ & $\begin{array}{l}\stackrel{\infty}{0} \\
\stackrel{\circ}{\circ}\end{array}$ & $\begin{array}{l}\stackrel{\infty}{P} \\
\stackrel{F}{F}\end{array}$ & $\begin{array}{l}\stackrel{\circ}{O} \\
\dot{+}\end{array}$ & $\frac{60}{60}$ & $\stackrel{\stackrel{\Xi}{O}}{E}$ & $\begin{array}{l}\stackrel{N}{0} \\
\stackrel{N}{\sim}\end{array}$ & $\begin{array}{l}\stackrel{2}{10} \\
\stackrel{0}{0}\end{array}$ & নু & $\begin{array}{l}\hat{0} \\
\stackrel{\sim}{\sim}\end{array}$ & $\begin{array}{l}\text { กิ } \\
\stackrel{0}{\circ}\end{array}$ & $\begin{array}{l}\stackrel{R}{2} \\
\stackrel{N}{\sim}\end{array}$ & $\stackrel{\text { Oִ }}{F}$ \\
\hline & & $\begin{array}{l}\frac{\Sigma}{\mathbb{N}} \\
\stackrel{\Phi}{\Sigma}\end{array}$ & $\begin{array}{l}8 \\
0 \\
o \\
+1 \\
+ \\
\dot{0} \\
\stackrel{N}{N}\end{array}$ & $\begin{array}{l}\hat{n} \\
\stackrel{n}{+1} \\
\text { N̦ } \\
\text { on }\end{array}$ & 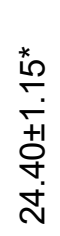 & 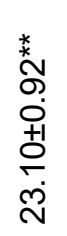 & 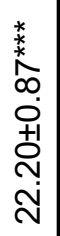 & 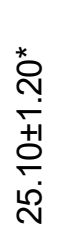 & 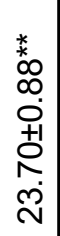 & 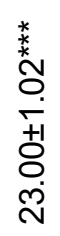 & 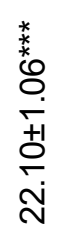 & 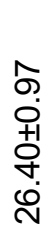 & $\begin{array}{l}\text { Oे } \\
\stackrel{+}{+1} \\
0 \\
\stackrel{0}{N}\end{array}$ & $\begin{array}{l}* \\
6 \\
\infty \\
0 \\
0 \\
01 \\
0 \\
\dot{0}\end{array}$ & 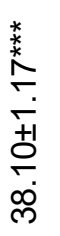 & 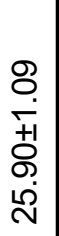 & \begin{tabular}{l}
$*$ \\
\multirow{2}{*}{} \\
0 \\
0 \\
+1 \\
+1 \\
$\infty$ \\
$\dot{N}$ \\
$\dot{N}$
\end{tabular} & 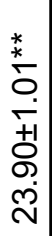 & 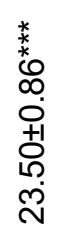 \\
\hline \multirow{2}{*}{ 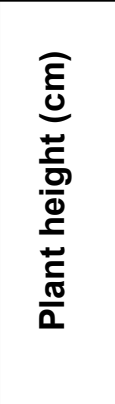 } & \multirow[b]{2}{*}{$N$} & गे & 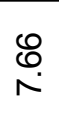 & 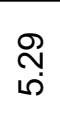 & $\begin{array}{l}\text { ¿ } \\
\end{array}$ & 8 & $\frac{\infty}{\sigma}$ & \begin{tabular}{l}
\multirow{0}{0}{} \\
0
\end{tabular} & $\begin{array}{l}\stackrel{6}{0} \\
\infty\end{array}$ & $\stackrel{R}{r}$ & $\stackrel{\grave{n}}{\wedge}$ & $\begin{array}{l}\infty \\
\stackrel{\infty}{\infty} \\
\infty\end{array}$ & $\frac{9}{\sigma}$ & $\begin{array}{l}\text { 8 } \\
\text { 움 }\end{array}$ & $\stackrel{m}{+}$ & $\begin{array}{l}n \\
\infty \\
\infty\end{array}$ & $\underset{\infty}{\curvearrowright}$ & \begin{tabular}{l}
0 \\
$ٌ$ \\
\hdashline
\end{tabular} & 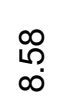 \\
\hline & & $\begin{array}{l}\text { ๘ँ } \\
\sum \\
\sum\end{array}$ & $\begin{array}{l}\stackrel{2}{N} \\
+ \\
+ \\
\stackrel{N}{N} \\
\stackrel{N}{N} \\
\stackrel{5}{\leftarrow}\end{array}$ & 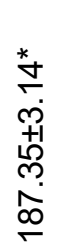 & 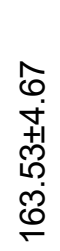 & 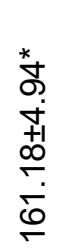 & 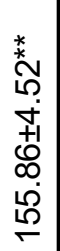 & 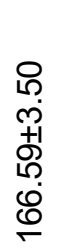 & 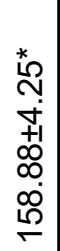 & 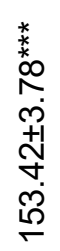 & 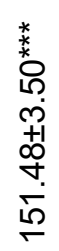 & 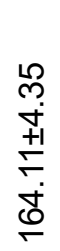 & 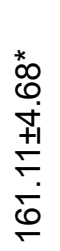 & 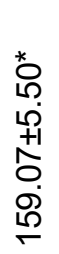 & 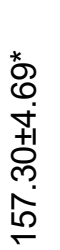 & 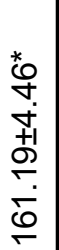 & 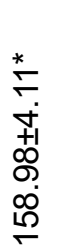 & 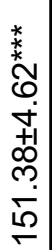 & 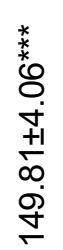 \\
\hline \multirow{2}{*}{ 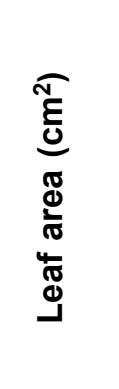 } & \multirow[b]{2}{*}{$\sigma$} & गे & 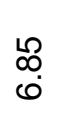 & $\stackrel{\Sigma}{\check{E}}$ & 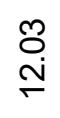 & $\begin{array}{l}\text { ऴ } \\
\stackrel{\circ}{\circ}\end{array}$ & $\begin{array}{l}\stackrel{m}{m} \\
\stackrel{\circ}{\circ}\end{array}$ & $\stackrel{\text { m }}{F}$ & $\begin{array}{l}\infty \\
\infty \\
0 \\
0\end{array}$ & $\begin{array}{l}\hat{\theta} \\
\dot{\nabla}\end{array}$ & $\begin{array}{l}\hat{\infty} \\
\infty \\
\infty\end{array}$ & $\begin{array}{l}\dot{0} \\
\infty \\
\infty\end{array}$ & 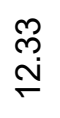 & กิ & مִ & $\stackrel{\stackrel{\mathscr{N}}{\rightleftharpoons}}{F}$ & $\begin{array}{l}\infty \\
\infty \\
\oplus \\
\oplus\end{array}$ & مُ & $\stackrel{\stackrel{N}{\sim}}{\stackrel{\sim}{\sim}}$ \\
\hline & & $\begin{array}{l}\text { 离 } \\
\stackrel{\Phi}{\Sigma}\end{array}$ & 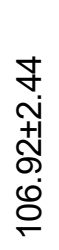 & 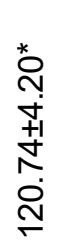 & 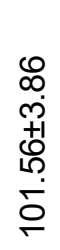 & 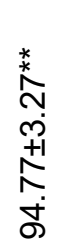 & 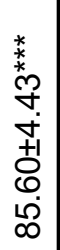 & $\begin{array}{l}0 \\
\stackrel{0}{0} \\
\oplus \\
1 \\
\infty \\
\infty \\
\dot{0} \\
\stackrel{0}{\sim}\end{array}$ & 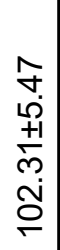 & $\begin{array}{l}\stackrel{*}{n} \\
\stackrel{m}{+} \\
\stackrel{+}{+} \\
\stackrel{+}{+} \\
\stackrel{m}{m}\end{array}$ & 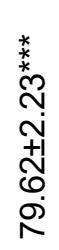 & 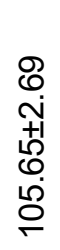 & $\begin{array}{l}\mathscr{8} \\
\ddot{1} \\
+1 \\
\stackrel{+1}{0} \\
\dot{0}\end{array}$ & 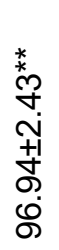 & 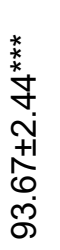 & 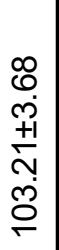 & 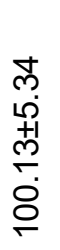 & 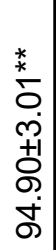 & 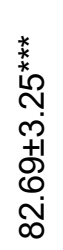 \\
\hline \multirow{2}{*}{ 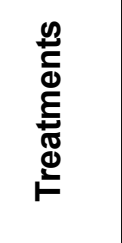 } & \multicolumn{2}{|l|}{ 芌 } & 0 & 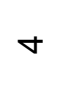 & $\theta$ & $\nabla$ & 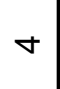 & $\infty$ & $\infty$ & $\infty$ & $\infty$ & $\theta$ & $\checkmark$ & $\nabla$ & $\nabla$ & $\infty$ & $\infty$ & $\infty$ & $\infty$ \\
\hline & \multicolumn{2}{|l|}{$\stackrel{\stackrel{0}{0}}{\overline{0}}$} & $\begin{array}{l}\overline{0} \\
\text { 은 } \\
\text { Oे }\end{array}$ & $\stackrel{20}{\sim}$ & প্ল & $\stackrel{1}{q}$ & 8 & $\stackrel{20}{\sim}$ & ৪ & $\stackrel{10}{+}$ & 8 & $r$ & $\sim$ & $m$ & $\forall$ & - & $\sim$ & $m$ & $\nabla$ \\
\hline \multicolumn{3}{|c|}{ suәбęnw } & & & & & $\sum_{\Psi}^{\infty}$ & & & & & & & & ๘ & & & & \\
\hline
\end{tabular}




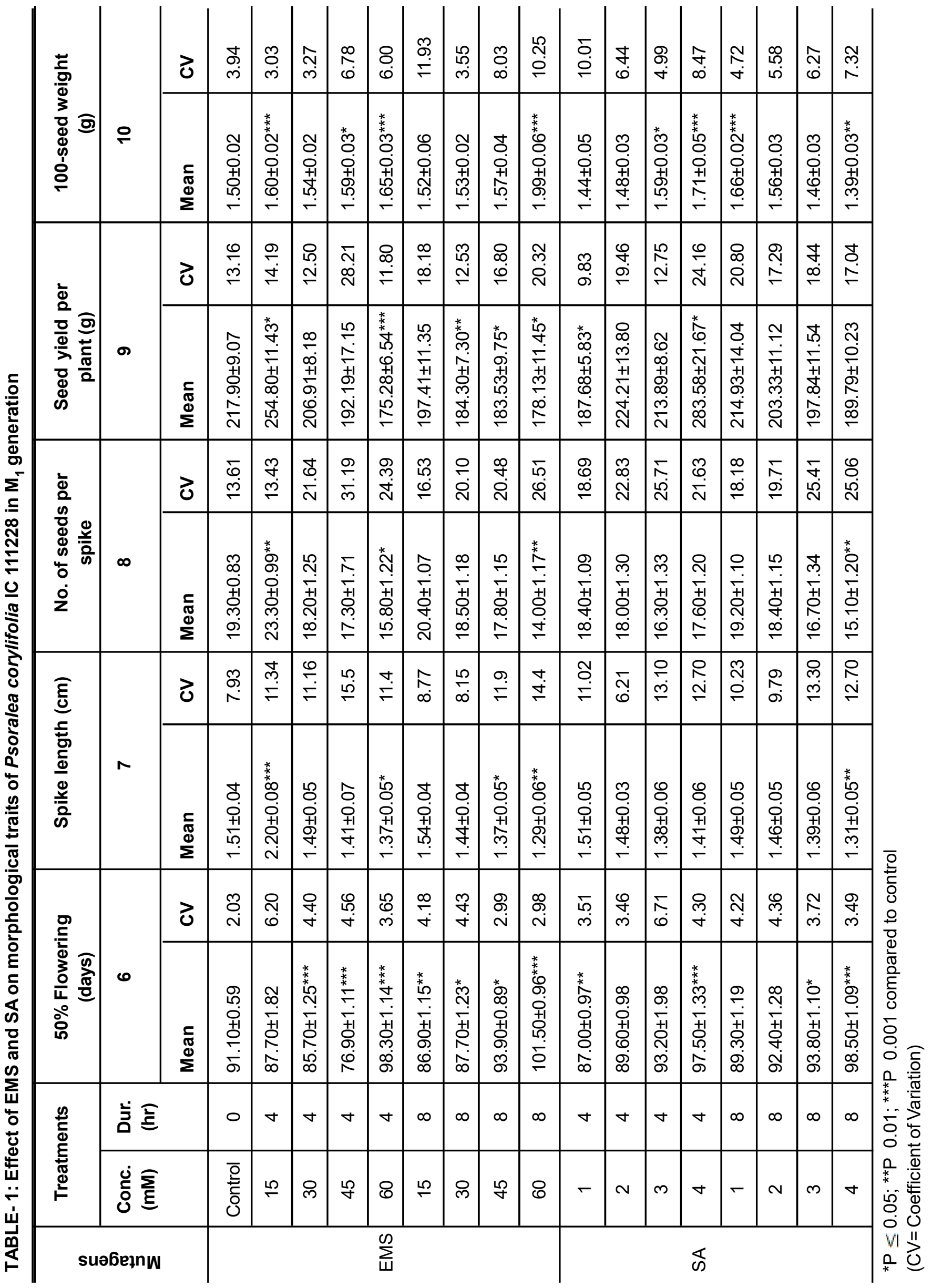




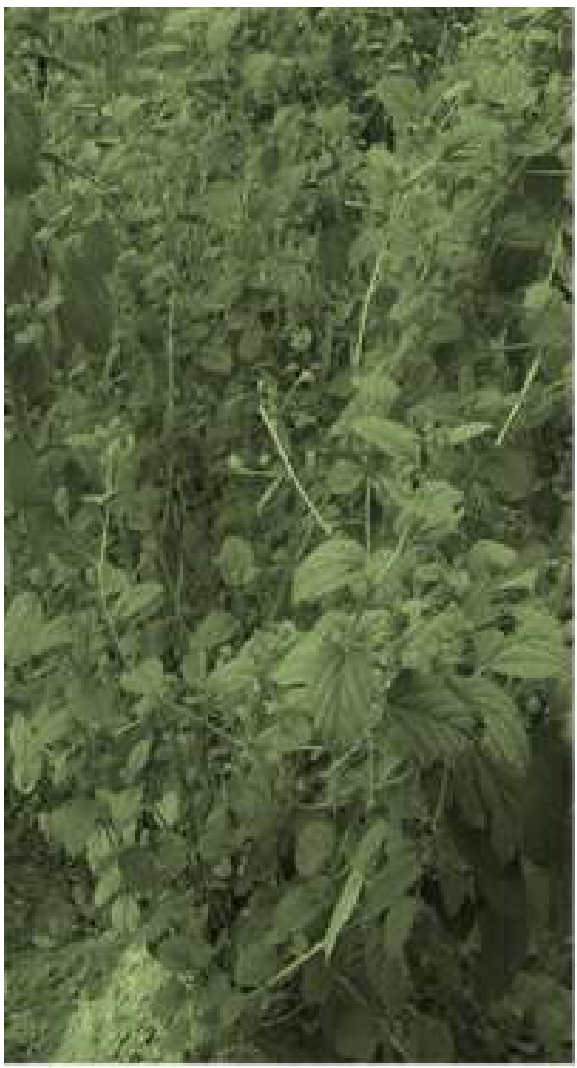

Control plants

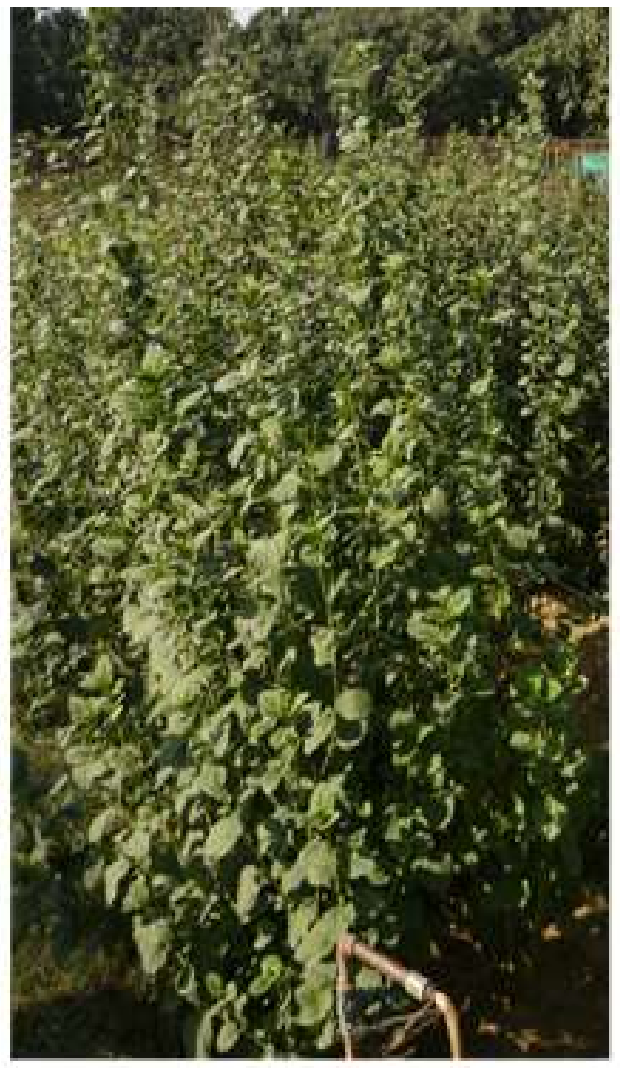

EMS treated plants

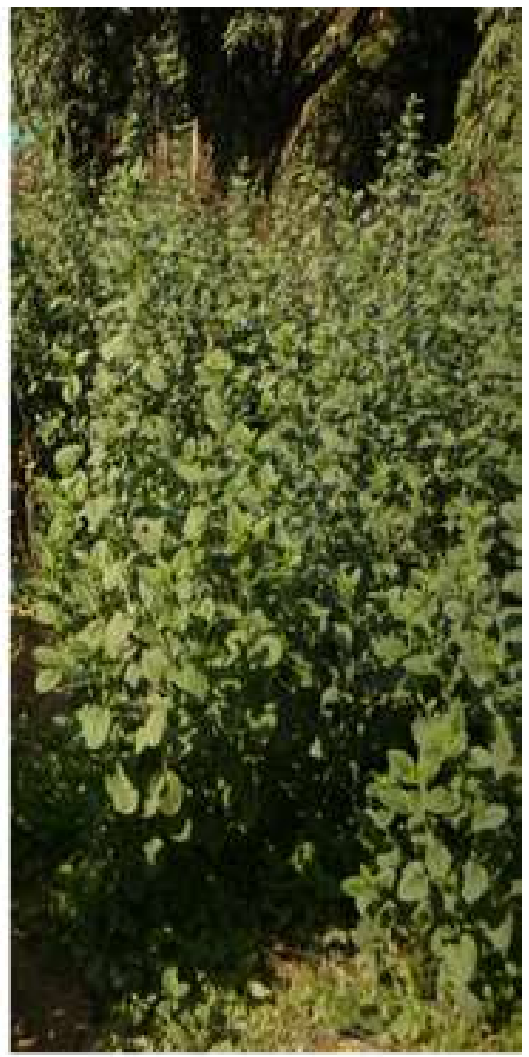

SA treated plants

Fig. 1: EMS and SA mutagens treated plants of Psoralea corylifolia IC 111228 in $M_{1}$ generation growing in the field

proved most effective in increasing total grain yield per plant and $15 \mathrm{mM}$ EMS applied for 4 hours increased the plant height in P. corylifolia IC 111228. The total yield per plant is a quantitative character and depends on a group of yield attributes (such as spike length, no. of seeds per spike, 100-seed weight, etc.), and the effect of mutagenic treatments on these characters give additive effect on total yield of the plants.

\section{References}

1. Chatterjee A, Shukla S, Mishra BK, Rastogi A, Singh SP. Induction of variability through mutagenesis in opium poppy (Papaver somniferum L.). Turk. J. Agric. For. 2012; 36(1):1-11.

2. Duke JA. Psoralea corylifolia seed extract has been found to stimulate the immune system, Econ. Bot. 1987; 41(4):524-526

3. Gaur PM, Gour VK, Srinivasan S. An induced brachytic mutant of chickpea and its possible use in ideotype breeding. Euphytica. 2008; 159(1):35-41.

4. Kondo Y, Kato A, Kubota Y, Nozoe S. Bakuchicin, a new simple furanocoumarin from Psoralea corylifolia. Heterocycles. 1990; 31(1):187-190.

5. Latha PG, Evans DA, Panikkar KR, Jayavardhanan KK. Immunomodulatory and antitumour properties of $P$ soralea corylifolia seeds. Fitoterapia. 2000; 71(3):223-231.

6. Prasad NR, Anandi C, Balasubramanian S, Pugalendi KV. Antidermatophytic activity of extracts from Psoralea corylifolia (Fabaceae) correlated with the presence of a flavonoid compound. J. Ethnopharmacol. 2004; 91(1): 21-24.

7. Siddiqui BA, Khan S. Breeding in Crop Plants: Mutations and in Vitro Mutation Breeding. $1^{\text {st }}$ Ed. Kalyani Publishers. 
Ludhiana. 1999.

8. Swaminathan MS. The detection of induced mutations, In: FAO/IAEA division of atomic energy in food and agriculture (ed). Manual on Mutation Breeding, International Atomic Energy Agency. Vienna. 1995; 138-141,

9. Wang $\mathrm{Y}$, Hong $\mathrm{C}, \mathrm{Zhou} \mathrm{C,} \mathrm{Xu} \mathrm{D,} \mathrm{Qu} \mathrm{H,} \mathrm{Cheng} \mathrm{Y.} \mathrm{Screening} \mathrm{antitumor} \mathrm{compounds} \mathrm{psoralen} \mathrm{and} \mathrm{isopsoralen} \mathrm{from}$ Psoralea corylifolia L seeds. Evid Based Complement Alternat. Med. 2009; 8(1): 1-7.

10. Wani AA, Anis M. Gamma ray- and EMS-induced bold-seeded high-yielding mutants in chickpea (Cicer arietinum L.) Turk. J. Biol. 2008; 32(3):161-166.

11. Xin D, Wang XDH, Yang J, Su YF, Fan GW, Wang YF, Zhu Y, Gao XM. Phytoestrogens from Psoralea corylifolia reveal estrogen receptor-subtype selectivity. Phytomed. 2010; 17(2): 126-131. 